\title{
Lo que suena en Gaztea Irratia. Origen, evolución e influencia social de la emisora musical pública vasca
}

\section{Gaztea Irratian entzuten dena. Euskal Herriko musika kate publikoaren} jatorria, bilakaera eta eragin soziala

\author{
What sounds in Gaztea Irratia. Beginning, evolution and social \\ influence of the Basque public music station
}

\author{
Ion Andoni Del Amo Castro* \\ Universidad del País Vasco (UPV/EHU)
}

\begin{abstract}
RESUMEN: Este trabajo explora las transformaciones e influencia social de la emisora musical pública vasca Gaztea Irratia, mediante la combinación de datos cuantitativos (audiencias), cualitativos (entrevistas), observación y análisis de contexto. La evolución muestra cómo ejerce de mediación entre la producción y escenas locales y los productos de la industrial cultural global. El cambio en 2006 hacia una radio-fórmula comercial ha sido polémico: los discursos legitimadores apelan al incremento de la audiencia, mientras los críticos, por contra, aducen la falta de compromiso con la cultura local. La influencia social, con todo, aparece al alza, incluso en los espacios festivos más alternativos.
\end{abstract}

PALABRAS CLAVE: EITB; jóvenes; lenguas minoritarias; Música; País Vasco; Radio.

ABSTRACT: This work explores the transformations and social influence of the public Basque music station Gaztea Irratia, through the combination of observation, context analysis, and qualitative (interviews) and quantitative (audiences) data. The evolution shows how it mediates between the products of the global cultural industry, and the local production and scenes. The change from 2006 towards a commercial radio-formula has been controversial: The legitimating discourses are justified in the increase of the audience; Critics, on the other hand, argue the lack of commitment to the local culture. Social influence, however, appears on the rise, even in the most alternative festive spaces.

KEYWORDS: EITB; Youth; Minority languages; Music; Radio; Basque Country.

\footnotetext{
* Correspondencia a / Corresponding author: Ion Andoni Del Amo Castro. Facultad de Educación de Bilbao, UPV/EHU, Barrio Sarriena, s/n (48940 Leioa) - https://orcid.org/0000-0003-0363-1636

Cómo citar / How to cite: Del Amo Castro, Ion Andoni (2019). «Lo que suena en Gaztea Irratia. Origen, evolución e influencia social de la emisora musical pública vascan, Zer, 25(47), 13-32. (https://doi.org/10.1387/zer.20619).

Recibido: 4 marzo, 2019; aceptado: 11 junio, 2019.

ISSN 1137-1102 - eISSN 1989-631X / (c) 2019 UPV/EHU

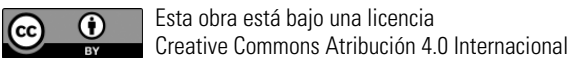




\section{Introducción}

El presente trabajo aborda el origen, evolución, transformación e influencia social de la emisora musical pública vasca Gaztea Irratia (inicialmente Euskadi Gaztea). Desde su creación en 1990 como Euskadi Gaztea, ha sido al tiempo registro e impulso de los cambios más recientes en la escena musical vasca. El giro de orientación a partir de 2006, hacia una concepción de radio-fórmula comercial juvenil, ha sido objeto de polémicas; se sintetizan aquí los discursos legitimadores y críticos en torno al mismo.

El artículo aborda un tema sobre el que no existe mucha bibliografia, tratando de fomentar una reflexión crítica en torno a la transformación de las emisoras públicas, sus contenidos y su papel socializador. Así, aborda la confluencia de música, juventud y transformación identitaria, en un territorio con cultura propia. El caso vasco resulta, al tiempo, especialmente interesante por la importancia de los movimientos musicales en las últimas décadas. En todo caso, consideramos que sus implicaciones teóricas podrían aplicarse igualmente a otros contextos, al menos a otros países del mundo occidental.

Comenzamos con un recorrido teórico por la discutida relación entre culturas juveniles, música, identidad, y medios de comunicación. A continuación, tras detallar la metodología, llevaremos a cabo una contextualización del caso vasco. Finalmente, se discuten las reflexiones en torno al cambio de Gaztea Irratia y su influencia social, para acabar sintetizando las principales conclusiones obtenidas.

\section{1. (Sub)Culturas juveniles y media}

La aparición de los medios de comunicación de masas (y de las propias masas) va a dar pie a un largo debate académico, político y social en torno a su papel (Martín-Barbero, 1993). En el ámbito más específico de las culturas juveniles y los media, la publicación en 1972 de la obra de Stanley Cohen «Folk Devils and Moral Panics» supone un hito significativo. En ella, el sociólogo describe la cobertura mediática de las subculturas juveniles Mods y Rockers en el Reino Unido en la década de 1960, acuñando el término de "pánico moral»: sobredimensionando un aspecto del comportamiento juvenil que suponía un desafio a las normas sociales existentes, los propios medios promovían más comportamiento socialmente «desviado».

Con todo, los trabajos de los investigadores reunidos en torno al Centre for Contemporary Cultural Studies (CCCS) de Birmingham, aportarían nuevas perspectivas, alejándose de las concepciones de la «desviación» y apoyándose en la concepción gramsciana de la hegemonía. Así, elaboran una teorización más dinámica sobre la relación entre los medios de comunicación de masas y las nuevas expresiones 
juveniles — subculturas - de la Gran Bretaña posterior a la segunda guerra mundial. En estas subculturas se produciría una «doble articulación», de un lado con la cultura paterna de clase trabajadora y, de otro, con la cultura dominante y los media (Hall y Jefferson, 2006; Hebdige, 2004).

Hebdige afirma que los medios no se limitan a registrar las subculturas, sino que las sitúan dentro del marco dominante de significados. Habla explícitamente de proceso de recuperación, mediante el cual «se repara el orden fracturado y la subcultura es integrada como entretenimiento dentro de la mitología dominante de la que en parte emana» (Hebdige, 2004: 130). De forma que:

se trata de sustituir una forma artística «al alcance de todas las conciencias» por "el cuento de hadas de la creatividad del artista», y un «ruido» (un caos de creación propia, con su coherencia y su lógica propias) por una «música» que será juzgada, despreciada o comercializada. (Hebdige, 2004: 137)

El concepto de subcultura autónoma desarrollado en los trabajos del CCCS, sin embargo, es objeto de una serie de críticas que se intensifican durante los 90 desde otros marcos teóricos, situados en la ola del postmodernismo, y agrupados como postsubculturalistas (Bennett, 2011; Muggleton, 2010). El trabajo de Sarah Thornton (1995) «Club Cultures», en torno a la música electrónica y la cultura dance, marca un hito significativo. Thornton propone más explícitamente que los medios ayudan desde el primer momento en la definición y construcción como subculturas de los movimientos incipientes. Desarrolla el concepto alternativo de "capital subcultural», que denotaría el estatus hip o cool que se acumula de legitimar los gustos underground propios a través de un proceso de distinción de aquellos valorados por el mainstream, a través de una continua clasificación y reclasificación.

Los trabajos post-subculturalistas que le siguen acentúan la importancia creciente de los medios y las industrias culturales en la configuración de los gustos e identidades juveniles (Muggleton, 2010). Así, las identidades juveniles se habrían vuelto más reflexivas, fluidas y fragmentadas debido a un creciente flujo de mercancías culturales, imágenes y textos a través de los cuales proyectos de identidad individualizados podrían ser «fashionados» (Bennett, 2011). La música todavía podría ser muy importante para algunos jóvenes como un medio de distinción social, pero la juventud mainstream estaría más inclinada a entrar y salir de la piscina de la música popular, en lugar de sumergirse en ella (Hesmondhalgh, 2005).

Con todo, una de las críticas más extendidas respecto de la teoría post-subcultural es que ésta adopta una ingenua y celebratoria posición con respecto al papel de las industrias culturales en la formación de las identidades, gustos y estilos de vida de la juventud (Hesmondhalgh, 2005). Con su énfasis en el consumo como práctica cultural clave en la juventud, la teoría post-subcultural adoptaría una postura esen- 
cialmente complaciente, que dibuja una juventud que se deja llevar en un supermercado del estilo, reduciendo la sensibilidad estilística a poco más que un juego de picar y mezclar, a una suerte de dandismo postmoderno.

Las últimas décadas, con todo, parecen apuntar a cambios en el significado social de la música (Del Amo, Letamendia y Diaux, 2016). Los cambios tecnológicos, que han individualizado y facilitado el acceso a la música, independizándolo en gran medida de las relaciones sociales, y de otra parte, la penetración de la propia lógica capitalista de usar y tirar, y la fragmentación cultural postmoderna, han modificado la relación con la música y su significado social. Antes que un elemento de construcción identitaria diferenciada, colectiva o más individualizada, lo que hoy primarían son sobre todo los elementos indiferenciadores, compartidos.

En un intento sintetizador, Bennett (2011) sostiene que una aproximación más fructífera en la investigación sobre la cultural juvenil debería permitirse mayor énfasis sobre la experiencia estructural en sí misma como reflexivamente manejada a través de la apropiación creativa de recursos culturales.

\section{Música, radio musical (pública) y nuevas tecnologías}

De la misma forma que industria y música se encuentran inseparablemente unidas y ambas se influencian entre sí (Frith, 2001), la retroalimentación entre radio y música (e industria musical) ha sido importante desde sus inicios, además de importante fuente de ingresos (Costa, 2016, 2017). La crisis del modelo radiodifusor americano ante la competencia de la televisión, junto a la implementación de la FM y la mejora técnica, propició a partir de los años 1950 la implantación de la especialización y las radio-fórmulas (Costa, 2013), dedicadas por completo a la música, y en muchos casos con vocación de prescriptores de discos. De una programación inicial basada en la música clásica, el auge del rock'n'roll dio paso al crecimiento de la radio musical y es en la confluencia entre los intereses de la industria discográfica y el público adolescente donde adquiere su legitimación la radio musical, combinando dos elementos, a saber, los contenidos y la identidad con la que se construye el relato radiofónico (Costa, 2013; Moreno, 1999; Keith, 1987). Todo ello ha situado a la radio musical en una posición hegemónica durante mucho tiempo (Costa, 2017).

En cuanto a los servicios públicos de radiodifusión, el propio concepto de servicio público ha sido difícil de delimitar, en tanto que negociado y permanentemente cuestionado, y tradicionalmente se ha relacionado a la radio de servicio público con la información, y no se incluye en ella a la radio musical, o, al menos, no de una manera explícita (Costa, 2016). La misión cultural, con todo, ha constituido una de las grandes justificaciones de los medios públicos, una suerte de renaissance del 
cultural remit, o un «¿quién si no nosotros?», que comprendería tres ámbitos: artístico e intelectual, identidad nacional, regional o lingüística, y diversidad cultural (Costa, 2016). Así, la música se asocia con la cultura, y ésta sería función de la radio pública musical. Además, el entretenimiento, aspecto clave de la radio musical, no está reñido con la cultura, por lo que debería unirse también a la idea de servicio público, y ahí se sitúa el caso Gaztea Irratia (Costa, 2016).

Es más, la contribución de la radio pública a la formación de escenas musicales ha sido también importante, como los casos de Radio 3 de Radio Nacional de España con la llamada "movida» (Fouce, 2008) o el papel de la británica BBC Radio 1 con el denominado britpop (Gilbert y Pearson, 2003). Con todo, la tensión entre la orientación cultural, entendida a menudo contrapuesta a lo comercial, y el entretenimiento, entendido como lo contrario, resulta constante, y remite al tiempo a la relación entre radio musical pública e industria musical (Costa, 2017), como a la dicotomía mainstream / underground o indie que señalaba Thornton (1995), y que enlaza también con los debates en torno a la autenticidad (Del Val, 2010).

El desarrollo de internet, con todo, ha abierto nuevas posibilidades. Autores como Jenkins (2008) señalan la emergencia de la cultura de la convergencia de los medios de comunicación, y de la cultura participativa, pasando del anterior rol separado entre productor y consumidor a otro en el que ambos interaccionan a través de un nuevo conjunto de reglas. Castells (2009) habla del paso de la comunicación de masas - unidireccional - a la autocomunicación de masas, en la que los sujetos pueden construir sus propias redes de comunicación, aunque las redes globales de empresas multimedia siguen ejerciendo un papel determinante.

Así, los servicios de música en streaming y las comunidades virtuales incluso intentan la adaptación del discurso radiofónico con la utilización de playlists o directamente lo que denominan "radios», que no son más que listas de reproducción sobre determinados artistas o géneros (Costa, 2013). A pesar de todo, el factor humano parece fundamental y, sobre todo, dificil de duplicar por los sistemas de recomendación digitales (Costa, 2013).

En todo caso, las facilidades para llegar a todo el mundo acaban por diluirse en un mar de sobreinformación (Zallo, 2011). Y es en este complejo y cambiante contexto, con una creciente interrelación entre medios de comunicación, nuevas tecnologías y culturas juveniles, en el que cabe analizar el papel actual de la radio musical.

\section{Metodología}

El presente estudio aborda el estudio de caso de la emisora musical pública Gaztea Irratia, en base a dos preguntas de investigación principales: 
- Cuál ha sido la evolución histórica y los cambios en Gaztea Irratia, así como los discursos legitimadores y críticos en torno a la misma.

— La influencia social de Gaztea Irratia, especialmente en los tiempos recientes.

A ellos se suma un objetivo de fondo más general que recorre el marco teórico:

- Reflexionar sobre las transformaciones en la relación entre música, juventud, identidad y medios de comunicación.

Consideramos que para responder a estas cuestiones el análisis cuantitativo de los datos de audiencia no resulta suficiente, y hemos de complementarlo con técnicas de investigación más cualitativas, combinadas con análisis de contexto. Esto es, lo que Griffin (2010) defiende como una lectura sintomática en el marco de un análisis coyuntural. Se trata de dar cuenta de la representación dinámica de la realidad realizada por los sujetos sociales; por lo que la interpretación sociológica de los discursos será un análisis contextual, donde los argumentos toman sentido en relación con los actores que los enuncian, enmarcados en un conjunto de fuerzas sociales en conflicto que los originan (Alonso, 2013).

La parte cualitativa se desarrolla en el marco de un estudio más amplio, que permite un análisis sincrónico y diacrónico más general de la relación entre Gaztea Irratia y los cambios socioculturales, haciendo énfasis en su influencia social. Así, se analizan extractos de entrevistas en profundidad que consideramos representativas, de un total de 43 entrevistas. Se distinguen tres tipologías básicas de entrevistas, que no son sino los actores principales de la escena musical y cultural:

— Músicos y Djs, tanto algunas figuras históricas como más jóvenes: 22 entrevistas.

- Programadores, tanto de locales comerciales de ocio, como culturales o autogestionarios: 10 entrevistas.

— Expertos, principalmente periodistas musicales, o activistas culturales: 11 entrevistas.

La selección ha partido del análisis bibliográfico, y de la técnica de la bola de nieve a partir de los primeros entrevistados. Las cuestiones en torno a Gaztea Irratia han formado parte del guión (semi-estructurado). Se han realizado principalmente a lo largo de 2014, que constituye un punto de referencia suficiente para evaluar la trayectoria de la emisora. El trabajo incorpora también observación directa en los espacios culturales y de ocio juveniles. A ello se suma la observación de los contenidos de la propia emisora, en el momento actual, así como la evocación de la trayectoria histórica a través de la memoria como oyente del propio investigador. 
En suma, se combinan datos cuantitativos, entrevistas, análisis de contexto, análisis de discurso, y observación directa. Sintetizamos a continuación las principales ideas que hemos obtenido, comenzando antes por una contextualización del escenario social y musical vasco.

\section{El contexto: la banda sonora vasca}

A partir de la década de los 60, las tierras vascas son testigo de hibridaciones de la cultura tradicional con nuevos fenómenos (contra)culturales, propiciando interesantes mutaciones. Todo ello se manifiesta especialmente en el campo de la música pop (Larrinaga, 2016).

El final de la década de 1960 y el comienzo de la de 1970 está atravesado por una fuerte conflictividad laboral, nacional y de lucha contra la Dictadura. En este contexto emerge la denominada Nueva Canción Vasca, en la estela de la modernización del folk, y en la que coinciden tres expresiones: la recuperación de la tradición y de la lengua, su movilización política, y los intentos de renovación estética (Amezaga, 1995). Los festivales o kantaldis alcanzan una importancia esencial; muchos de ellos eran reivindicativos (anti-nucleares, a favor del euskara o pro amnistía) constituyendo un pretexto para que la gente se reuniera (Larrinaga, 2016).

Los ayuntamientos, al tiempo, van cediendo competencias en la organización de las fiestas a comisiones populares, con presencia de grupos culturales, deportivos, políticos, y anti-represivos. Para conseguir financiación instalan tabernas temporales, txosnas, en las calles, que desarrollan también una oferta musical propia, y que se convierten en espacios efimeros pero influyentes (Larrinaga, 2016).

Finalizando la década de 1970 parece constatarse el agotamiento de un ciclo cultural. La década de 1980, sin embargo, está marcada por un potente estallido punk, etiquetado como Rock Radikal Vasco (RRV). Sus territorios serán fundamentalmente los de clase obrera e inmigración española de los 50-60, con el castellano como lengua dominante. Pero difícilmente puede explicarse sin el humus de antagonismo desarrollado en el periodo anterior y el contexto del momento. El punk vasco acumula así un doble rechazo: a las consecuencias excluyentes de la crisis económica, y a las consecuencias, excluyentes y represivas, de la reforma política del Régimen español (Larrinaga, 2016; Sáenz de Viguera, 2007). Y acometerá una prolífica praxis de construcción de canales propios de información, okupaciones de Gaztetxes (casas de jóvenes), y resignificación de espacios (Del Amo, 2016). De especial importancia resulta la proliferación de radios libres que permiten la difusión de las inquietudes sociales, políticas, culturales y musicales que no encontraban hueco en los medios convencionales (Diaux, Del Amo, y Letamendia, 2017; Santos, 1999). 
Los 90 supondrán la consolidación de una contracultura, principalmente en euskera a partir de 1988 (Amezaga, 1995), que engrana en torno al rock vasco una conjunción que une idioma, estética y mensaje. Jóvenes socializados en esta (contra) cultura aparen en las calles, colegios, conciertos, txosnas y gaztetxes; se consolida una nueva identidad cultural (Larrinaga, 2016).

En torno al final de siglo, sin embargo, parecen producirse cambios importantes. Algunos de carácter general a las sociedades occidentales, que reflejan los trabajos post-subculturalistas. En el caso vasco, además, se suman los efectos de los procesos de institucionalización, que propician la rutinización de la identidad y cultura euskaldun, que se perciben garantizadas (CEIC, 2005). La emisora musical pública vasca Euskadi Gaztea resulta testigo de todos estos cambios.

\section{El cambio de Gaztea Irratia}

En el marco del debate sobre la autonomía, se fortalecieron también las demandas de una radio propia y, en general, de un espacio comunicativo vasco. La respuesta administrativa a estas reivindicaciones llegó en 1982, cuando el Gobierno Vasco, en base al reciente Estatuto de Autonomía de Gernika de 1979, creó el grupo de radio-televisión pública Euskal Irrati Telebista, EITB. Ese mismo año comenzó sus emisiones de radio Euskadi Irratia, en euskara, y al año siguiente Radio Euskadi, inicialmente bilingüe y posteriormente en castellano a partir de 1986 (Gutiérrez, 2002).

Cuando las emisoras generalistas del grupo público vasco se encaminaban hacia su décimo aniversario, EITB decidió poner en marcha una emisora musical dirigida al público joven. No resultaba una apuesta sencilla, pues «Los 40 principales» de la Cadena SER gozaba de amplia implantación; debía, por tanto, ofrecer algo nuevo (Gutiérrez, 2002). El 21 de marzo de 1990 comienza sus emisiones Euskadi Gaztea (Euskadi joven), en castellano desde Bilbao y en euskara desde Donostia. Esta duplicidad no acabaría de cuajar, y en 1994 se elimina la emisión en castellano, manteniéndose únicamente en euskara. La cercanía y el idioma constituyen, pues, su oferta diferencial, consiguiendo atraer a una masa de jóvenes vasco parlantes entre 16 y 20 años, e incluso a quienes no lo son, al tratarse de una fórmula sencilla que puede seguirse también sin conocer bien el idioma. A partir de 1996, además, otorga especial protagonismo a programas ligeros conducidos por locutores jóvenes y con participación de los oyentes, como «Hi bizi haiz»y «Salgai» (Gutiérrez, 2002).

Siguiendo esa característica diferencial de cercanía, inicialmente la emisora dará voz a muchos de los grupos de rock vascos del momento, que habitualmente poblarán su lista de éxitos. De hecho, éstos grupos, hasta entonces, casi únicamente habían encontrado eco en la red de radios libres locales y en la emisora de la izquierda inde- 
pendentista, Egin Irratia, a la que Garzón decidió bajarle el volumen en 1998 (Sáenz de Viguera, 2007).

Este carácter alternativo inicial cambiaría en el nuevo milenio con la adopción de la radiofórmula de éxitos contemporáneos (Contemporary Hit Radio), en las horas centrales de su programación, basada en temas actuales, más comercial, y enfocada al público más juvenil. En cualquier caso, mantiene — defienden sus responsablesprogramas de orientación más alternativa en la franja nocturna, siguiendo la conocida sentencia de Frances Line, director de BBC Radio 2 durante los ochenta, «ratings by day, reputation by night», muy ligado al easy y el hard listening, que sitúa los gustos musicales al margen del mainstream en una franja programática marginal (Costa, 2013).

Tras su cambio de registro a partir de 2006 Gaztea Irratia es (auto)descrita profusamente como una «radio fórmula juvenil», aumentan las alusiones al entretenimiento, y a una función de servicio público centrada en la promoción del euskara (Costa, 2017). Contrasta esta definición, con todo, con el caso de Catalunya (iCat.Cat), donde se habla en términos de promoción de la escena musical catalana (Costa, 2017).

Una de sus figuras referentes desde el comienzo, Joseina Etxeberria, refleja el discurso legitimador del cambio hacia una fórmula más comercial, que considera la ha situado entre las más escuchadas, y en euskara:

Bai egia da Gaztearen ibilbide musikalean aldaketa bat izan dela. Duela 9 urte inguru hartutako erabaki bati erantzuten dio aldaketa honek. Kritika batzuk sortu dituen aldaketa, baina entzule kopuru hazkunde nabarmen bat ere bai. Urteetan geunden 70 mila entzuleetan estankatuta eta egun 130/140 mila entzulengana iristen gara. $\mathrm{Ni}$, orain ez nago arlo musikalean, azken 7 urte hauetan entertainment-ean ari naiz... Baina erabat defendatzen dut aldaketa musikal hori, eta egia esan behar bada, nik askoz lehenagotik eskatzen nuen aldaketa komertzial hori. Gainontzeko medio guztiek bezalaxe, guztiek eh!, Gazteak ere diru publiko bat jasotzen du, baina beste zati handi bat publizitatetik jasotzen du... Krisi garai honetan publiak nabarmen egin du behera medio guztietan, bai eta dirulaguntza publikoak ere... Nik ez dakit gaur egun, Gaztea garai bateko zenbakiekin posible izango ote zen... Musikalki? Komertzialtasun batean mugitzen gara, baina Europa osoa mugitzen den komertzialtasun batean. Gaur egun Gaztean entzuten den musika, Londreseko edo Paris edo Berlingo edozein irrati komertzialetan entzun liteken musika bera da. Musika horrek, Euskal Herriko 4. irratirik entzunena izatera eraman gaitu. Baina irrati guztien artean eh! Euskaraz arituta!!! Euskaraz egindako irrati batek inoiz ez du halako zenbakirik eman. 40 Principalesi Gipuzkoan 20 mila entzuleko aldea ateratzen diogu, ez Araba eta Bizkaian, baina bi probintzia horietan ere ikaragarrizko gorakada izan dugu. Hemengo 
musikan eraginik izan duen? Bai, ziurrenik bai... Orain maketa lehiaketan Enkore, Skasti edo Belako bezalako taldeak saritzen ditugu. Duela urte gutxi EHn ez zen halako musikarik egiten ${ }^{1}$. (Joseina Etxeberria, periodista de Gaztea, entrevista personal)

En la misma línea, el director de Gaztea Irratia, Jon Lamarka, remarca en el estudio de Lola Costa (2017: 66) el empleo de la música comercial como «elemento netamente relacionado con el entretenimiento más juvenil» y fruto de una decisión directamente relacionada con la audiencia: «la gran crisis de Gaztea fue en el año 2006 y ahí se estudió que nos guste o no las minorías las dejas aparte y no tenemos presupuesto para todo». El carácter «glocal» es una seña de identidad de Gaztea Irratia a día de hoy, que inserta en su programación los productos de la industria cultural global, en un gran porcentaje en inglés (Elordui, 2015).

Aunque hay quienes admiten que sigue siendo mejor que otras, las críticas hacia su carácter de radio-fórmula repetitiva, sin embargo, son generalizadas entre los entrevistados del presente estudio. Los discursos críticos contrastan la fórmula actual con los inicios más alternativos. También se le reprocha falta de compromiso con la escena vasca. Desde el entorno autogestionario de los gaztetxes, se critica también que se exija estar en la SGAE para participar en su popular concurso de talentos «Maketa Lehiaketa». Algunas opiniones acusan incluso censura para con aquellos estilos que se alejan del canon comercial y easy listening, aspecto que Joseina Etxeberria llega a admitir:

Beno, saiatzen gara diskrimizazio positibo bat egiten hemengo taldeekin. Badakigulako, hemengo irrati bat, euskaraz eta hemengo musikari tarte bat

\footnotetext{
1 Sí, es cierto que ha habido un cambio en la trayectoria musical de Gaztea. Este cambio responde a una decisión tomada hace alrededor de 9 años. El cambio ha generado algunas críticas, pero también un aumento considerable de la cantidad de oyentes. Llevábamos años «estancados» en 70.000 oyentes y hoy en día llegamos a los 130.000-140.000 oyentes. Yo ahora no estoy en la sección musical, en los últimos 7 años estoy en el entertainment... Pero defiendo absolutamente ese cambio, y a decir verdad, yo pedía desde mucho antes ese cambio comercial. Como todos los demás medios, ¡todos eh! Gaztea también recibe dinero público, pero otra parte importante la recibe de la publicidad... En estos tiempos de crisis la publicidad ha bajado notablemente en todos los medios, y también las ayudas publicas... Yo no sé si hoy día Gaztea sería posible con los números de otros tiempos... ¿Musicalmente? Nos movemos en una comercialidad, pero en una comercialidad en la que se mueve toda Europa. La música que se escucha hoy en día en Gaztea es la misma música que se podría escuchar en cualquier radio comercial de Londres, París o Berlín. Esa música nos ha llevado a ser la cuarta radio más escuchada de Euskal Herria. ¡Pero entre todas las radios, eh! ¡Y en euskara! Una radio hecha en euskara jamás ha dado semejantes números. A los 40 Principales les sacamos una diferencia de 20.000 oyentes en Gipuzkoa, no en Bizkaia y Araba, pero en esas dos provincias también hemos tenido una subida espectacular. ¿Si ha tenido influencia en la música de aquí? Pues, seguramente sí... Actualmente en el concurso de maketas [«Maketa Lehiaketa»] premiamos grupos como Enkore, Skasti o Belako. Hace unos pocos años en Euskal Herria no se hacia semejante música.
} 
eginez egin behar dugula. Hala omen diote irrati honen estatutuek, eta guk ere hala nahi dugu gainera. Hemen egindako musika asko gustatzen zaigu, nahiz eta badakigun denek irrati komertzial batean ez dutela kabidarik. Horren inguruan zentsuratzaileak garela eta horrelako hitzak bota izan dizkigute... Gazteentzako irrati komertzial batean, adibide batzuk jartzearren, Benito Lertxundi, Amaia Zubiria edo Anestesiak badute kabidarik? Ez dakit... Gure irratia leku publiko askotan jartzen dute, eta hala jarraitzea nahi genuke, eta zentzu horretan easy listening puntu bat behar du. Lertxundi eta Zubiria entzuteko Euskadi Irratia dago EiTBko irratien artean, eta Anestesia entzuteko bere garaian Gazteak bazituen eta baditu, iluntze partean, saio berezi batzuk. Normalean musika horiek ez ditugu formulan sartzen... Baina bai Berri Txarrak, Gatibu, Esne Beltza... bezalako taldeak. Europako zein irrati komertzialetan entzuten da, formularen barruan, horrelako musika? ${ }^{2}$ (Joseina Etxeberria, periodista de Gaztea, entrevista personal)

EiTB Musika, la emisora de «hilo musical» del mismo grupo, definida como una radio con contenidos específicos de cultura y música de calidad (Costa, 2017; Gutiérrez, 2002), es una referencia citada en positivo por los entrevistados. Otra también señalada en los discursos críticos es la emisora musical estatal Radio 3, junto a las radios libres o las radios temáticas on line. El veterano Dj Makala añade además la $B B C$ y sugiere la idea de poner en marcha una nueva emisora musical pública que dé espacio a fórmulas menos comerciales.

El propio Dj Makala, que tiene un programa sobre músicas del mundo en Gaztea Irratia, admite que determinados estilos no se trabajan mucho en tierras vascas, precisamente aquellos más pop admitidos en la radio-fórmula, lo que provoca la sensación de repetición y de que siempre están sonando los mismos. Por contra, otras críticas reprochan que se opte de manera fácil y automática por los productos de la gran industria musical en lugar de apoyar lo que se hace «aquí», donde también, por

2 Bueno, intentamos hacer una discriminación positiva con los grupos de aquí. Porque sabemos que, una radio de aquí, tenemos que hacerla en euskara y haciendo un hueco a las y los músicos de aquí. Así lo dicen los estatutos de esta radio, y además nosotros y nosotras también lo queremos así. Nos gusta mucha de la música hecha aquí, aunque sabemos que todos no tienen cabida en una radio comercial. Al respecto de eso, nos han acusado de que somos censuradores y cosas similares... En una radio comercial juvenil, ¿tienen cabida, por poner unos ejemplos, Benito Lertxundi, Amaia Zubiria o Anestesia [cantautor folk, cantante clásica y grupo hardcore-thrash metal]? No sé... Nuestra radio se pone en muchos sitios públicos, y quisiéramos que siguiera siendo así, y en ese sentido necesita un punto easy listening. Para escuchar a Lertxundi y Zubiria ya está Euskadi Irratia entre las radios del grupo EiTB, y para escuchar a Anestesia Gaztea ya tenía en su momento, y tiene, en la franja nocturna, programas especiales. Normalmente no metemos esos tipos de música en la radio-fórmula... Pero sí grupos como Berri Txarrak, Gatibu, Esne Beltza... ¿En qué radio comercial de Europa se escucha, dentro de la radio-fórmula, semejante música? 
ejemplo, se hace electrónica o rap. El_Txef_A, uno de los productores y Djs vascos con mayor proyección internacional, apela a que «si quieres ser un país, tío, curra como un país», "aquí es como a medias todo». Una crítica adicional apunta a la falta de compromiso con la formación y educación musical que supone la adopción masiva de la radio-fórmula comercial, más tratándose de una emisora pública. Los responsables de Gaztea Irratia admiten en gran medida estas críticas:

Para una emisora orientada a la música comercial como es Gaztea Irratia, el encaje programático de los músicos desconocidos resulta complicado. $\mathrm{Su}$ director señala la apuesta por dar una oportunidad pero a aquellos grupos que ya se han dado a conocer en cierta medida, a veces en su propio concurso de talentos, para lo que destaca el papel de la programadora musical en esta tarea: «A ver, es una relación dificil. Normalmente los grupos que están sonando en euskera o locales es porque ya han demostrado algo, o bien porque han ganado algún premio, «Maketa Lehiaketa» o bien porque tienen muchas visitas en Internet.» (Costa, 2017: 67)

Por otro lado, la emisora ha trabajado desde sus inicios la interacción con el público, en algunos programas y, especialmente en la elaboración de su lista de éxitos «Top Gaztea» a través de votación de la audiencia, o en eventos off line como el concurso de talentos "Maketa Lehiaketa", que culmina con actuaciones en directo en la fase final. Ha incorporado también un concurso de Djs, que va por su tercera edición, y que de igual modo finaliza con un evento en directo. La interacción con el público en directo se produce también a través del apoyo y cobertura de la emisora a los conciertos breves del espacio Ahotsenea durante la multitudinaria feria del libro y disco de Durango, o en torno al macro-festibal BBK Live de Bilbao. Algunos conciertos en directo o falso directo, sobre todo en formato acústico, completan la relación de la emisora con la música en directo, quizás no tan potente como en otras emisoras como Radio 3, pero existente.

En los últimos años, al igual que el conjunto del grupo EITB, también se está esforzando en estrategias transmedia y de convergencia (Elordui, 2015). La web y las redes sociales constituyen la referencia principal en ese camino: en programas como «DidaGaztea» la interacción con el público a través de las redes sociales constituye un núcleo central, y la propia web aloja videoclips, playlists, vídeos de conciertos y productos de la industria cultural global, pero también productos audiovisuales generados por el público en secciones como «Parody», «Destination EH» o «Youtuberrak» (Elordui, 2015).

El registro de utilización del euskara también ha sufrido cambios — no exentos de críticas - pasando de un diseño sociolingüístico normalizador basado en el euskara batua (unificado) estándar, hacia un diseño heteroglósico y de vernaculización que trata de conectar con las locuciones y prácticas socio-estilísticas juveniles (Elor- 
dui, 2016). Esto sucede en la emisión de radio, mientras que el lenguaje escrito en la web o redes sociales prima el euskara batua estándar, utilizando el cambio de código al castellano o inglés para conseguir el estilo informal buscado por la emisora (Elordui, 2015). En los contenidos generados por los usuarios, sin embargo, se refleja tanto la importante presencia de lenguajes vernáculos y dialectos locales, como de bricolaje socio-lingüístico que mezcla otros idiomas como el castellano e inglés (Elordui, 2015).

\section{Lo que suena en Gaztea Irratia: la influencia social}

Los datos de audiencia han sido uno de los elementos argumentales principales en el discurso legitimador de los cambios. En efecto, el histórico de audiencia de los últimos años (Gráfico 1) registra que la emisora despega de los 70.000 oyentes en torno a los cuales Joseina Etxeberria la calificaba de "estancada», alcanza los 96.600 oyentes en 2009 y se sitúa por encima a partir de entonces, entre los 110.000 y 120.000 , con un pico de 143.600 oyentes en 2011 .

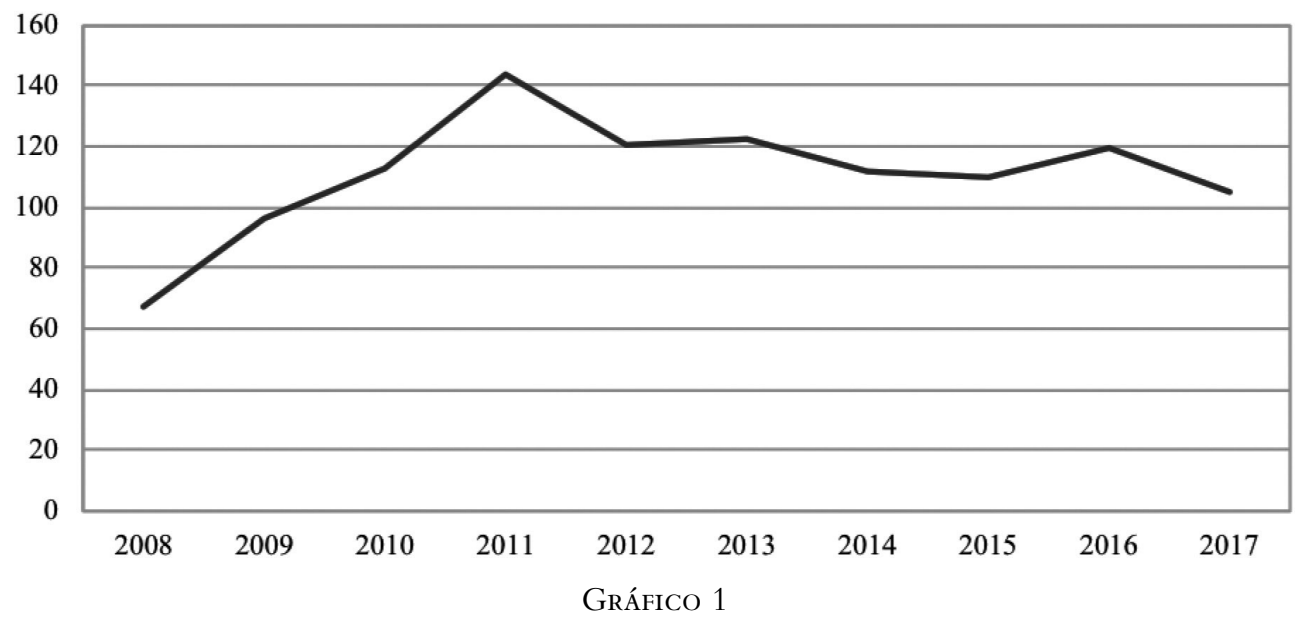

Evolución de la audiencia de Gaztea Irratia (miles de oyentes)

Según la medición del CIES acumulada en 2016, Gaztea Irratia se sitúa en Euskadi en 109.000 oyentes, por delante de competidoras como Cadena 40 (88.000 oyentes), Cadena 100 (77.000), o Europa FM (47.000). En Nafarroa en 2017, con una posición más discreta, registra una audiencia según CIES de 11.000 oyentes, frente a 19.000 de Cadena 40, 23.000 de Rock FM, 33.000 de Europa FM, y 43.000 de Cadena 100. Según la oleada de febrero a noviembre de 2018 del Estudio General de Medios (EGM), Gaztea Irratia se sitúa en los 82.000 oyentes de lunes a domingo, y 88.00 de lunes a viernes. 
Para Euskadi y Nafarroa en conjunto en 2017, el desglose según sexo, edad, idioma y territorio queda registrado en el Gráfico 2. La radiografía de la audiencia refleja que se concentra especialmente en Bizkaia y Gipuzkoa (que también son los territorios más poblados), con un perfil ligeramente más femenino, fundamentalmente euskaldun, capaz de entender, leer y escribir en euskara, y especialmente entre 25 y 34 años.

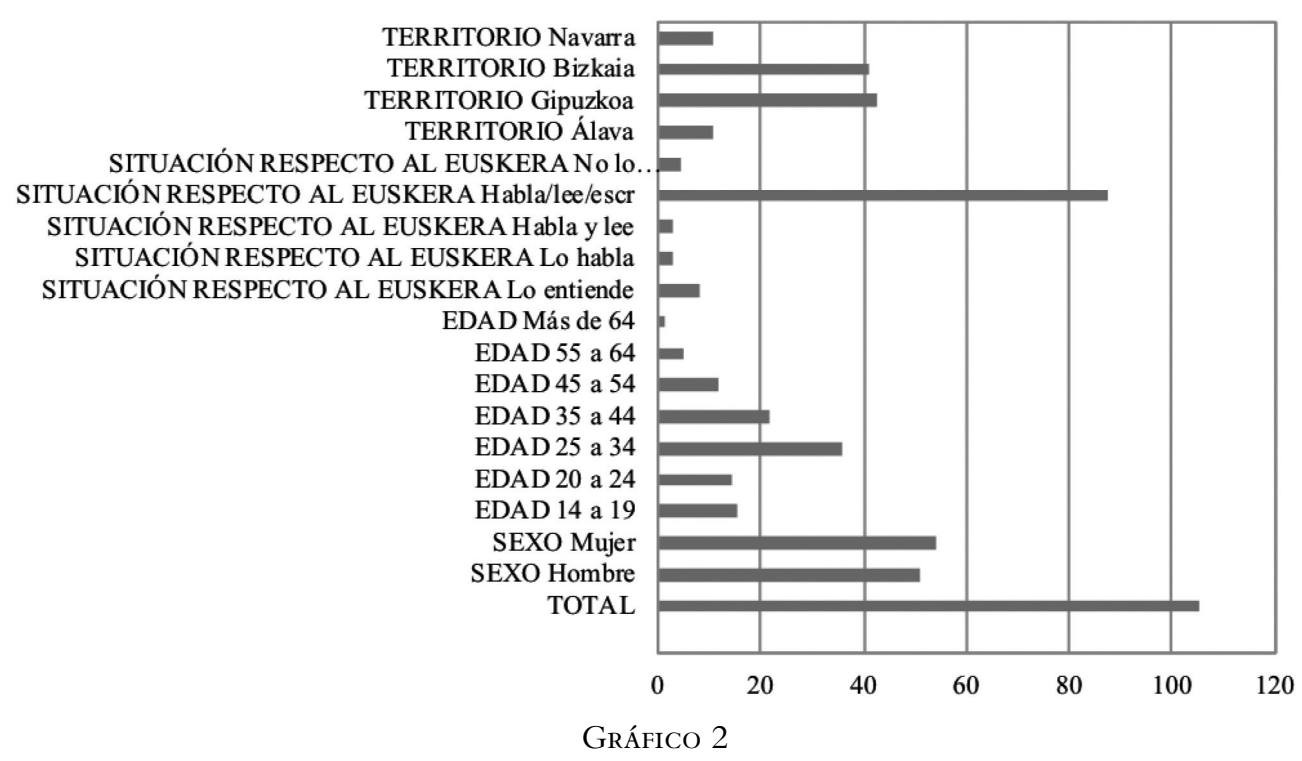

Estructura de la audiencia de Gaztea Irratia (miles de oyentes)

Pero más allá de la constatación de la consolidación de los datos de audiencia que constituyen el argumentario de los discursos legitimadores del cambio, el trabajo de investigación del que parte este artículo permite explorar también la influencia social de Gaztea Irratia de modo cualitativo. Y de forma especialmente interesante en los espacios de ocio alternativos y populares que vienen de la década de 1980.

Así, las y los entrevistados reconocen que los conciertos en directo en los bares prácticamente han desaparecido, aunque parecen recuperarse aquellos de pequeño formato o acústicos y en horarios más vespertinos, especialmente en zonas como la comarca de Pamplona. En algunos casos han sido sustituidos por Djs; pero son los menos. La lista de reproducción — a menudo reflejando la radio-fórmula de Gaztea Irratia - es lo que se habría generalizado.

Ello ocurre incluso - y significativamente- en los espacios festivos articulados en base a las txosnas organizadas por grupos culturales, sociales y políticos, que durante las décadas de 1980 y 1990 habían llegado a (auto)caracterizarse como «es- 
pacios temporalmente autónomos» (Sáenz de Viguera, 2007), marcados sobre todo por el rock y una importante presencia del euskara. Las txosnas de Bilbao, en plena disputa con el Ayuntamiento que intenta impulsar otros espacios, constituyen la vanguardia de un cambio que pretende - con notable éxito- salvar el espacio. Así, a distintos ritmos según las particularidades locales, y aunque la música en directo se mantiene, durante los últimos años el cambio fundamental se da cuando, en la música grabada, las vertientes más comerciales entran también masivamente en las txosnas. ¡Hasta el reggaetón! (Miner, 2013).

La selección de la música en las txosnas, relatan los miembros de la comisión de txosnas de Mungia, se realiza a menudo en base a la lista de éxitos «Top Gaztea»:

- Lista egiteko erabili genuen Gazteako Top 10a edo... Top 20 edo dago Gaztean, hortik ateratzen dituzu 20 horiek...

- Eta denak dira erderaz edo ingelesez... ${ }^{3}$

(Miembros de la comisión de txosnas de Mungia, Bizkaia, entrevista personal)

La influencia de Gaztea Irratia se registra también en el que es el formato de evento musical (y social) al alza: el festival. Incluso en aquellos de orientación más alternativa o reivindicativa. Kalera Rock en Durango (Bizkaia) es uno de éstos, a favor de los derechos de los presos vascos de motivación política. Con un formato invernal (en torno a febrero), y un horario fundamentalmente diurno que atrae a un público muy joven, reconvertido en 40 Minutu Rock celebró su séptima edición en 2019. Y pivotando fundamentalmente en torno al rock, aunque en la edición de 2018 incorporó Djs como Las Tea Partys. Joseba, uno de sus organizadores confirma que los grupos con mayor tirón son los de Gaztea Irratia, los que en ese momento se encuentran en su lista «Top Gaztea»:

Arrakastatsuenak dira Euskadi Gazteakoak, bai, bai... Beno hori izena ipini diot jakiteko zeintzuk diren, baina klaro, bai, «Top Gaztean» daudenak. Eta nik uste dut pasa den urteko jaialdiaren arrakasta horregatik izan zela, justu «Top Gaztean» zeudelako jaialdiko lau edo bost talde 4 . (Joseba, festival «Kalera Rock», entrevista personal)

\footnotetext{
3 - Para hacer la lista utilizamos el Top 10 o así de Gaztea... Hay un Top 20 o así en Gaztea, y de ahí sacas esos $20 \ldots$

- Y todos son en castellano o inglés...

${ }^{4}$ Los más exitosos son los de Euskadi Gaztea, sí, sí... Bueno, les he puesto ese nombre para saber cuáles son, pero claro, sí, los que están en el «Top Gaztea». Y yo creo que el éxito del festival del pasado año fue por eso, porque justo cuatro o cinco grupos del festival estaban en el «Top Gaztea».
} 
En todos estos espacios, lo que suena en Gaztea Irratia constituye una referencia. Y los entrevistados aducen una razón principal: que permite compartir, que resultan canciones conocidas o «famosas» para cantar juntos. Se registra así también una primacía de lo que Mejías y Rodriguez (2003) señalan como elementos indiferenciadores en la música. Y al tiempo, una influencia creciente de la industria cultural global que apuntaban los estudios postsubculturalistas; son estos productos (fundamentalmente en inglés o castellano) los que alimentan en mayor medida la música programada por Gaztea Irratia en sus franjas centrales, tras el cambio hacia una orientación más comercial. Las producciones de la audiencia que tienen cabida en la web de Gaztea Irratia reflejan también esa primacía de retorno: un ejemplo claro lo constituyen los videoclips elegidos por los jóvenes para parodiarlos en la sección «Parody» de la web de Gaztea Irratia, casi en su totalidad éxitos internacionales en inglés adaptados por los jóvenes al contexto local (Elordui, 2015).

El proceso de hibridación (hacia lo comercial) de Gaztea Irratia se reproduce, pues, también incluso en los espacios más alternativos que venían de las décadas anteriores. La apertura, en general, se considera positiva por los entrevistados, pero al tiempo, parece implicar cierta disolución, pérdida de profundidad e intensidad, e implica una dosis importante (e interesante) de ambigüedad:

Zabalpen batek, adibidez, eta estetikan bariedade gehiago dela, ba estetika horren atzean dagoena ezagutu behar duzu. Ez dakizu? Lehen ikusita bakarrik bazenekien; orain ikustea ez da nahikoa, orain jakin egin behar duzu estetika horren atzean ze ideia dagoen. Eta hor dago anbiguedade hori, estetika jakin baten atzean egon ahal da, ez dakit, ba ideia borrokalari batzuk, pixka bat filosofia hori, baina igoal igoal egon ahal da ba Euskadi Gaztearen fan amorratu bat ${ }^{5}$. (Oliba Gorriak, grupo rock, entrevista personal)

A Gaztea Irratia, en gran medida como pretendía, se le atribuye una caracterización más liviana y festiva, opuesta a la caracterización más combativa de buena parte de la música vasca de décadas anteriores. Ha definido también un modelo social y estético. Y también otra forma de relación con la música, que como apunta David Hesmondhalgh (2005), estaría más inclinada a entrar y salir en la piscina de la música popular, que a sumergirse en ella.

\footnotetext{
5 Hay una apertura, en la estética hay más variedad, y tienes que conocer lo que hay detrás de esa estética. ¿No sabes? Antes solo con ver ya sabías; ahora ver ya no es suficiente, ahora tienes que saber que ideas hay detrás de esa estética. Y ahí está la ambigüedad: detrás de una estética concreta puede haber, no sé, pues unas ideas combativas, un poco esa filosofia, pero igualmente puede estar un aguerrido fan de Euskadi Gaztea.
} 


\section{Conclusiones}

La emisora pública Gaztea Irratia, desde su nacimiento en 1990 como Euskadi Gaztea, ha sido al tiempo registro e impulsora de los cambios en el significado social de la música en tierras vascas. Su creación tiene lugar en un contexto en el que la música popular había adquirido una gran importancia, en términos culturales, sociales, de renovación estética, y de movilización política, desde la Nueva Canción Vasca de las décadas de 1960 y 1970, al estallido punk y rock de la década de 1980. Estos movimientos desarrollan un alto grado de autonomía, al margen de los grandes medios de comunicación. Es más, especialmente durante la década de 1980, desarrollan sus propios circuitos de comunicación social alternativa, con una gran importancia de las radios libres.

En su origen en este contexto, Euskadi Gaztea se comporta como un altavoz de la producción musical del país, algo que hasta el momento prácticamente solo realizaban los medios alternativos. Y precisamente esto, junto al idioma, le proporcionan su principal ventaja competitiva: la cercanía. Podría establecerse como hipótesis para otra investigación, además, en qué medida la creación de Euskadi Gaztea contribuyó a la crisis de las radios libres en la primera mitad de los 90.

Una vez asentada en torno a los 70.000 oyentes con estos postulados iniciales, el cambio hacia una orientación más comercial en 2006 pudiera interpretarse como un alejamiento de esa característica de cercanía y una asunción de los productos de la industria cultural global. Sin embargo, Gaztea Irratia parece conectar también con cambios que están sucediendo entre la juventud vasca: el relativamente rápido crecimiento de audiencia, que casi llega a doblar, no puede explicarse únicamente como un efecto de dominación mediática. Los estudios internacionales sobre culturas juveniles, en efecto, ya venían registrando una creciente importancia de los productos de la gran industria cultural global en las construcciones de gustos e identitarias. El discurso legitimador de los cambios utiliza, precisamente, ambos argumentarios: los datos de audiencia y las referencias internacionales ("como cualquier otra radio comercial»).

Tras el cambio de registro, la influencia social de Gaztea Irratia parece haber aumentado en los últimos años. Lo muestran los datos de audiencia, pero también otros cualitativos. Tanto los propios espacios que Gaztea Irratia ofrece a las producciones de sus oyentes como, sobre todo, los testimonios recogidos en las entrevistas, apuntan a su creciente influencia en contenidos musicales, culturales y estéticos. Incluso, y de forma especialmente significativa, en los espacios festivos más autónomos y alternativos que venían de las décadas anteriores.

La influencia de los productos de la industria cultural global aparece, así, crecientemente importante entre la juventud vasca. Lo hace, en efecto, entrelazada con 
la experiencia y las estructuras locales. Y entre éstas destaca el papel de Gaztea Irratia, porque esas producciones globales aparecen mediadas por la emisora musical pública: sobre todo son influyentes aquellas que suenan en ella.

Todo ello parece apuntar también a fenómenos más de fondo en torno al propio significado social de la música. Los entrevistados subrayan que para los jóvenes importa no tanto el género, sino que la canción sea conocida, para poder cantar juntos. Una relación con la música más liviana, festiva y compartida, un entrar y salir de la piscina de la música popular. Así, antes que un elemento de construcción identitaria diferenciada, colectiva o más individualizada, lo que hoy prima es la sociabilidad, compartir, cantar juntos. En un contexto de fragmentación social y cultural, además del aspecto lúdico, de pasarlo bien, que siempre ha sido parte determinante, el sensorium con el que conecta, lo que la música proporciona hoy en día es, sobre todo, indiferenciación, una experiencia compartida, el placer de cantar en multitud.

En ese contexto, las emisoras musicales encuentran un renovado impulso en su labor de prescripción: pueden hacer que determinadas canciones resulten conocidas para ser compartidas. Y en el caso vasco tiene una referencia reiterada: la gente quiere lo que suena en Gaztea Irratia.

Todo el proceso de cambio de Gaztea Irratia, con todo, no ha resultado exento de críticas. A pesar de su creciente influencia social, o precisamente por ello. Los discursos críticos le reprochan ejercicios de censura, la primacía otorgada a los productos de la gran industria cultural global, falta de impulso de la escena local y de compromiso con la formación musical. De hecho, su propia definición sitúa el focus sobre el entretenimiento y el impulso del idioma, resolviendo así en el idioma su compromiso cultural como emisora pública, frente a otras posibles definiciones que podrían primar la promoción de la escena musical local. Los discursos normativos críticos, sin embargo, apelan a un compromiso público más integral, que haga también mayor incidencia en la cultura propia y la formación.

Se pide, en suma, que en Gaztea Irratia suenen también otras cosas, una mayor conexión cultural. Lo cual no hace sino reiterar la importancia social de Gaztea Irratia y lo que suena en ella.

\section{Referencias bibliográficas}

Alonso, L. E. (2013). La sociohermenéutica como programa de investigación en sociología. ARBOR Ciencia, Pensamiento y Cultura, 189 (761). doi: http://dx.doi.org/10.3989/ arbor.2013.761n3003

Amezaga, J. (1995). Herri kultura: Euskal Kultura eta Kultura popularrak. Leioa: UPV/EHU. 
Bennett, A. (2011). The post-subcultural turn: some reflections 10 years on. Journal of Youth Studies, 14(5), 493-506. doi: https://doi.org/10.1080/13676261.2011.559216

Castells, M. (2009). Comunicación y poder. Madrid: Alianza.

CEIC (2005). Hacia una nueva cultura de la identidad y la política. Tendencias en la juventud vasca. Vitoria-Gasteiz: Servicio Central de Publicaciones del Gobierno Vasco.

Cohen, S. (1972). Folk Devils and Moral Panics. Londres: MacGibbon \& Kee.

Costa, L. (2013). Como lo oyes. La publicación de listados de reproducción de los programas temáticos musicales en la radio de titularidad pública en España. Trípodos, 33, 73-98.

Costa, L. (2016). Música y significado. Análisis de los términos asociados a la radio musical en España en los documentos legales y corporativos desde la perspectiva del servicio público. Derecom, 20, 153-172.

Costa, L. (2017). ¿Indie vs. mainstream? Una aproximación a la relación entre industria musical y radio musical pública en España. Revista de la Asociación Española de Investigación de la Comunicación, 4(8), 63-72. doi: http://dx.doi.org/10.24137/raeic.4.8.8

Del Amo, I. A. (2016). Party \& Borroka. Jóvenes, músicas y conflictos en Euskal Herria. Tafalla: Txalaparta.

Del Amo, I. A., Letamendia, A. y Diaux, J. (2016). ¿El declive del significado social de la música?. Revista Crítica de Ciências Sociais, 109, 11-32. doi: http://dx.doi.org/10.4000/rccs.6189

Del Val, F. (2010). El Canto del Loco: El debate sobre la autenticidad del rock. En J. Noya, F. del Val y C. M. Pérez Colman (eds.), Musyca. Música, sociedad y creatividad artística (pp. 145-157). Madrid: Biblioteca Nueva.

Diaux, J., del Amo, I.A. y Letamendia, A. (2017). Freedom Waves: Giving People a Voice and Turning It Up! Tuning into the Free Radio Network in the Basque Country. Westminster Papers in Communication and Culture, 12(2), 59-81. doi: http://doi. org $/ 10.16997 /$ wpcc. 228

Elordui, A. (2015). Diseinu linguistikoa eta transmedia-engaiatzea: Gaztea, audientziari begira prosumitzaileen aroan. En A. Elordui y E. Arana (eds.), Transmedia Komunikazio estrategiak. Gaurkotasun-edukiak diseinatzeko eta hedatzeko bide berriak (pp. 135-154). Bilbo: Udako Euskal Unibertsitatea.

Elordui, A. (2016). Elebakartasunetik heteroglosiara: Gaztea Irratia, bernakulizazioa gaztekomunikabideen diseinu sozioestilistikoan. Uztaro, 97, 31-52.

Fouce, H. (2008). Emociones en lugar de soluciones. Música popular, intelectuales y cambio político en la España de la Transición. TRANS Revista Transcultural de música, 12.

Frith, S. (2001). The Popular Music Industry. En S. Frith y W. Strax (eds.), Pop and Rock (pp 26-52). Cambridge: Cambridge University Pres,

Gilbert, J. y Pearson, E. (2003). Cultura y políticas de la música dance. Disco, hip-hop, house, techno, drum'n'bass y garaje. Barcelona: Paidós Comunicación.

Griffin, C. E. (2010). The trouble with class: researching youth, class and culture beyond the 'Birmingham School'. Journal of Youth Studies, 14(3), 245-259. doi: https://doi.org/10. 1080/13676261.2010.533757 
Gutiérrez, A. (2002). Euskal irratigintzaren historia. Bilbo: Udako Euskal Unibertsitatea.

Hall, S., \& Jefferson, T. (eds.). (2006). Resistance Through Rituals: Youth Subcultures in Post-war Britain. London, New York: Routledge.

Hebdige, D. (2004). Subcultura. El significado del estilo. Barcelona: Paidós.

Hesmondhalgh, D. (2005). Subcultures, Scens or Tribes? None of the Above. Journal of Youth Studies, 8(1), 21-40. doi: https://doi.org/10.1080/13676260500063652

Jenkins, H. (2008). La cultura de la convergencia de los medios de comunicación. Barcelona: Paidós.

Keith, M.C. (1987). Radio Programming: Consultancy and Formatics. Boston: Focal Press.

Larrinaga, J. (2016). Euskal musika kosmikoak. Euskal musika popularra gizartearen isla eta aldatzailea. Mungia: Baga-biga.

Martín Barbero, J. (1993). De los medios a las mediaciones. Comunicación, cultura y hegemonía. México: GG Mass media.

Megías, I. y Rodríguez, E. (2003). Jóvenes entre sonidos. Hábitos, gustos y referentes musicales. Madrid: INJUVE.

Miner, K. (2013). Papito eta mamitak txosnetan. Naiz / Gara, 20 de septiembre.

Moreno, E. (1999). La radio de formato musical: concepto y elementos fundamentales. Comunicación y Sociedad, 12(1), 89-111.

Muggleton, D. (2010). From classlessness to clubculture. A geneaology of post-war British youth cultural analysis. Young, 12(2), 205-19.

Sáenz de Viguera, L. (2007). Dena ongi dabil! ¡Todo va dabuten! Tensión y heterogeneidad de la cultura radical vasca en el límite del estado democrático (1978-...). (Tesis doctoral). Recuperado de http://dukespace.lib.duke.edu/dspace/handle/10161/390

Santos, M. T. (1999). La Radio Vasca (1978-1998). Bilbao: Servicio editorial de la UPV/ EHU.

Thornton, S. (1995). Club Cultures: Music, Media and Subcultural Capital. Cambridge: Polity.

Zallo, R. (2011). Civilización y vida social. Paradojas de la cultura digital. Revista TELOS (Cuadernos de Comunicación e Innovación), 88, Julio-Septiembre. 